\title{
Learning by Explaining Orally or In Written Form? Text Difficulty Matters
}

\author{
Leonie Jacob ${ }^{1}$, Andreas Lachner ${ }^{2,1}$, Katharina Scheiter ${ }^{1,2}$ \\ ${ }^{1}$ Leibniz-Institut für Wissensmedien, Tübingen, Germany \\ ${ }^{2}$ University of Tübingen, Germany
}

Correspondence concerning this article should be addressed to Leonie Jacob, Leibniz-Institut für Wissensmedien, Tübingen, Schleichstraße 6, D-72076 Tübingen. E-mail: 1.jacob@iwmtuebingen.de. Phone: $+49(0) 7071-979-284$.

\section{Acknowledgement}

We would like to thank Eleonora Dolderer, Maike Köncke, and Anna Rosenträger for their assistance in conducting the study and rating the qualitative data. Moreover, we would like to thank Christian Burkhart and Zarah Weiß for providing us with the computer-based text difficulty measures. The research reported in this article was supported by the Federal Ministry of Education and Research in Germany (BMBF) under contract number 01JA1611.

Leonie Jacob is a doctoral student at the LEAD Graduate School \& Research Network [GSC1028], which is funded within the framework of the Excellence Initiative of the German federal and state governments. 


\begin{abstract}
In this experiment, we examined whether text difficulty moderates the effect of the modality of explaining on students' learning. Students $(N=115)$ read a high-difficult and a low-difficult text. Additionally, students generated either a written or an oral explanation. A control group of students retrieved the content. For the low-difficult text, we found no significant differences between conditions. For the high-difficult text, however, oral explaining yielded better comprehension than writing explanations. The retrieval condition showed the lowest performance. Mediation analyses revealed that the effect of explaining modality was mediated by the number of personal references and the comprehensiveness of the generated explanations. Our findings suggest that the effect of explaining modality emerges when students are required to learn from difficult text materials. Furthermore, the findings show that oral explaining is effective, as it triggers distinct generative processes due to increased social presence during explaining.
\end{abstract}

Keywords: learning by explaining, text comprehension, modality effect, learning by teaching, generative learning 


\section{Introduction}

Learning from texts is a prevalent learning activity at all levels of education. Therefore, students are required to select the relevant information from the text, to organize and elaborate the selected information to gain a deep understanding (Wharton \& Kintsch, 1991). Throughout these cognitive processes, students have to monitor their understanding to be able to detect and resolve potential comprehension problems (Kiewra, 2005). Despite the ubiquitous use of text materials, research indicates that students often face difficulties in realizing these strategies spontaneously which may constrain their general comprehension (O'Reilly \& McNamara, 2007). Therefore, the question remains which additional learning activities support students' learning from texts. Generating explanations of the learning contents (to fictitious others) is such an activity (Fukaya, 2013; Lachner, Backfisch, Hoogerheide, van Gog, \& Renkl, 2019; Roscoe, 2014; Roscoe \& Chi, 2008). Explaining triggers distinct cognitive (Fiorella \& Mayer, 2014) and metacognitive processes (Fukaya, 2013; Lachner et al., 2019) which contribute to students' deep comprehension and their monitoring accuracy. In his meta-analysis, Kobayashi (2018) documented the beneficial effects of explaining. Results indicated a medium effect of explaining to fictitious others on students' comprehension ( $g=0.48$, based on 28 comparisons). However, the heterogeneity among the studies was high, indicating that additional factors constrained the effectiveness of explaining. Against this heterogeneity of empirical evidence, the sparse number of empirical studies investigating potential boundary conditions of explaining is surprising (Hoogerheide, Deijkers, Loyens, Heijltjes, \& van Gog, 2016; Lachner, Ly, \& Nückles, 2018 for exceptions). Recently, researchers started to investigate moderation effects of the modality of explaining, that is generating oral or written explanations (Hoogerheide et al., 2016; Lachner, Ly, \& Nückles, 2018), however, only produced mixed findings. They either demonstrated that oral explaining was superior to writing explanations or showed that generating oral and written 
explanations was comparably effective. In this study, we argue that the modality of explaining is not a boundary condition per se, but rather depends on the difficulty of the text material which is used during the study phase. Such effects can be assumed, as previous research on text comprehension highlighted the crucial role of text difficulty features (O'Reilly \& McNamara, 2007; Ozuru, Briner, Best, \& McNamara, 2010). Furthermore, recent research demonstrated that the effectiveness of distinct consolidation activities depends on the quality of the previous study phases and students' constructed mental representations (e.g., Lachner et al., 2019; Roelle \& Nückles, 2019). The current study contributes to a better understanding of whether and how the modality of explaining may support students' learning, depending on the difficulty of the text material of the pre-given study phase.

\subsection{Learning by Explaining}

Explaining is a consolidation activity which has primarily been used in interactive learning situations in which students either generate explanations to present to peers (Palinscar \& Brown, 1984; Plötzner, Dillenbourg, Praier, \& Traum, 1999; Roscoe, 2014; Roscoe \& Chi, 2008) or to pedagogical agents in computer-supported environments (Chin, Dohmen, Cheng, Oppezzo, Chase, \& Schwartz, 2010; Okita \& Schwartz, 2013). However, even without such interactions explaining is still an effective strategy, as documented by recent research in which students provided explanations to a fictitious other student (Fiorella \& Mayer, 2013, 2014; Hoogerheide et al., 2016; Hoogerheide, Visee, Lachner, \& van Gog, 2019).

For instance, Fiorella and Mayer (2014, Experiment 2) examined the effects of preparing to explain the learning contents versus preparing and explaining the contents to fictitious others on students' learning. First, university students received an expository text about the Doppler effect and were informed that they would answer a test (test expectancy) or that they would explain the learning contents to a fictitious student (explaining expectancy) after the study phase. Afterwards, 
half of the students either restudied the text (no teaching condition) or provided an explanation on video (explaining condition). The authors found that explaining expectancy only improved students' short-term retention, (teaching expectancy effect: $d=0.55$ ) but that the actual act of explaining was particularly beneficial for long-term retention (explaining effect: $d=0.56$ ). Hoogerheide, Loyens, and van Gog (2014, Experiment 1) asked students to learn from an instructional text on syllogistic reasoning. Two groups were required either to prepare for a test or to prepare to explain the learning materials. A third group of students prepared to explain, and additionally explained the learning contents on video. As in the study by Fiorella and Mayer (2014), students who provided an explanation outperformed students who only prepared to explain $(d=0.75)$. The findings demonstrate that the act of generating explanations enhanced students' comprehension.

\subsection{The Modality of Explaining Affects Students' Learning}

Recently, researchers aimed at replicating the effects of learning by explaining in written form, as writing explanations can be more easily implemented in educational practice and may be a more feasible instructional approach during teaching. For instance, Hoogerheide et al. (2016, Experiment 1) used the identical learning materials from their previous study (Hoogerheide et al., 2014), and investigated potential effects of the intention to provide an explanation or to actually write an explanation. Contrary to their previous findings, neither the intention to provide an explanation nor writing an explanation affected students' learning (see Fukaya, 2013, for related findings). In their second experiment, the authors directly compared written versus oral explaining, and additionally included a control condition in which students simply restudied the learning material. Results indicated that explaining on video $(d=0.43)$ but not writing an explanation $(d=0.19)$ was more effective than restudying. However, there were no significant differences between the written and oral condition $(d=0.24)$. The authors supposed that 
generating oral but not written explanations triggered students' awareness of social presence, which inclined them in more generative processes, such as reorganizing and elaborating the content toward the need of potential recipients (Chafe \& Tannen, 1987). Additional explorative content analyses showed that students in the oral explaining condition used more personal references, as an indicator of a social presence, such as "me", and "you" than students who wrote an explanation $(d=1.54)$. Apparently, oral explaining triggered higher levels of social presence; however, this did not result in a higher learning performance.

In their replication study, Lachner et al. (2018) provided students with a Wikipedia entry on combustion engines as learning material. Afterwards, the students either generated an oral or written explanation. Contrary to Hoogerheide et al. (2016), the authors found that students who explained orally outperformed students who wrote an explanation $(d=0.67)$. Additional analyses showed that students who generated an oral explanation, again used more personal references $(d$ $=0.98$ ), indicating higher levels of social presence. At the same time, they found that the higher test performance of students in the oral explaining condition was explained by the higher levels of generative processes, as students provided more elaborations in their explanations $(d=1.53)$. The authors suggested that the differences between their study and the study by Hoogerheide et al. (2016, Experiment 2) can be ascribed to the different learning materials. Whereas Hoogerheide et al. (2016) used well-designed instructional material which likely can be characterized by low levels of text difficulty, Lachner et al. (2018) used a naturalistic expository text from Wikipedia with likely higher levels of text difficulty, which may account for the contradictory findings. Following these suggestions, although oral explaining may generally trigger higher levels of social presence regardless of the level of text difficulty, it may be superior to writing explanations only when students are required to learn from high-difficult text material (as in the study by Lachner et al., 2018). When learning from low-difficult text material (as in the 
study by Hoogerheide et al., 2016), the different explaining modalities may be comparably effective, as students may have ample cognitive resources available to generate an appropriate representation of the text (Ozuru, Dempsey, \& McNamara, 2009). When learning from highdifficult texts, additional learning activities such as oral explaining may be effective to trigger distinct generative process (e.g., elaborations) that aim at building a coherent situation model of the contents.

\subsection{Text Difficulty Affects Students' Learning}

Following McNamara (2013), text difficulty can be described as a multi-dimensional construct comprising different text features, that is syntactic complexity, concreteness, and cohesion of a text.

Syntactic complexity refers to the difficulty of the phrase structure and the associated dependencies within a text (Berendes et al., 2018). For instance, complex syntactical structures comprise the use of nominals, or the inclusion of subordinate clauses. This often unnecessarily increases the sentence length, and as such increases readers' cognitive efforts to process the text (Berendes et al., 2018). Contrarily, concreteness refers to the use of content words that are concrete, meaningful, and evoke mental images which make texts easier to process and comprehend (McNamara, 2013). Finally, cohesion refers to explicit indicators that mark distinct relations between sentences (e.g., connectives, argument overlap) to process adjacent sentences as meaningful units (Ozuru et al., 2010). Thus, during processing expository texts, cohesion mainly helps students establish a coherent initial understanding because they highlight interrelations among adjacent sentences. Research indicated that text difficulty features, such as cohesion or concreteness, play a distinct role in comprehending texts (Hinds, Patterson, \& Pfeffer, 2001; Lachner \& Nückles, 2015; McNamara, 2013; McNamara, Kintsch, Songer, \& 
Kintsch, 1996; McNamara \& Knitsch, 2009). In this study, we therefore used text difficulty (i.e., cohesion, concreteness) to manipulate our learning material.

\subsection{The Present Study: Effects of Explaining Modality and Text Difficulty on Students' Learning}

Based on the previous considerations, we conducted an experiment in which we investigated effects of explaining modality (i.e., generating written versus oral explanations) and text difficulty (high-difficult versus low-difficult text), and potential interaction effects on students' learning. For this purpose, we provided university students with two different texts from Golke and Wittwer (2018) which systematically differed regarding text difficulty (i.e., level of cohesion and word concreteness, see McNamara, 2013). Additionally, as a subsequent learning activity, the students explained the contents of the learning materials either in written or oral form. A control condition retrieved the contents of the learning material. Retrieving material is an effective and robust strategy to enhance learning (c.f., testing effect, Roediger \& Karpicke, 2006), and therefore, can be regarded as a strong control condition in comparison to explaining.

We stated the following hypotheses (which were preregistered on As.Predicted, https://aspredicted.org/sv3zv.pdf).

1.4.1 Learning-outcome hypotheses. Regarding effects of text difficulty, we hypothesized that students should gain higher test scores when learning from the low-difficult text than from the high-difficult text. Regarding effects of explaining modality, based on the contradictory findings by Hoogerheide et al. (2016) and Lachner et al. (2018), we hypothesized a moderation effect of text difficulty: For the high-difficult text, generating an oral explanation should be more beneficial than generating a written explanation (Lachner et al., 2018). This effect can be assumed, as the induced generative processing during oral explaining should contribute to students' text comprehension, as they may help to construct a more coherent mental 
representation of the text. Students who only retrieved the contents of the learning material (i.e., retrieval practice) should show the lowest performance on the knowledge test. For the lowdifficult text, we assumed that students who provide an explanation (i.e., in written or oral form) should outperform students in the retrieval condition. The effect of the explaining modality should be less pronounced in the low-difficult text condition, as students would have ample cognitive resources available to build an adequate representation of the text which should result in comparable learning gains of the two explaining conditions.

1.4.2 Additional explorative analyses. Regarding the underlying processes which account for the differences within students' learning performance in the high-difficult text condition, we analyzed the quality of the generated explanations. Based on previous research (Chafe \& Tannen, 1987; Hoogerheide et al., 2016; Lachner et al., 2018), we assumed that oral explaining would increase the awareness of social presence, as indicated by more personal references in the explanations. The increased awareness of social presence would incline students to provide more comprehensive explanations (as measured by the number of explanatory concepts, Boshuizen $\&$ Schmidt, 1992) and more elaborations (Chafe \& Tannen, 1987; Lachner et al., 2018) to make the content tangible to a fictitious novice student. These generative processes should finally contribute to students' comprehension.

As further dependent variables, we collected subjective ratings of students' mental effort and difficulty as potential indicators of their perceived cognitive load. Additionally, since generating explanations has demonstrated to result in higher levels of students' monitoring accuracy (Fukaya, 2013; Lachner et al., 2019), we further collected students' meta-comprehension ratings, as proxies for their meta-comprehension accuracy.

\section{Method}

\subsection{Participants and Design}


Students $(N=119)$ from non-biology study programs of a German university participated in the study. We excluded data from four students, because they did not complete the study $(n=3)$ or reached very low performance (i.e., zero points) in the reading skill test $(n=1)$.

The mean age of the students was 25 years $(S D=7.76)$ and $77 \%$ of them were female. The students were on average in their $5^{\text {th }}$ semester $(S D=3.72)$. Most students were enrolled in humanity programs $(n=89)$.

We conducted a $2 \times 3$ mixed factorial design with text difficulty as a within-students factor (low-difficult text vs. high-difficult text) and learning activity as a between-students factor (generating oral vs. written explanations vs. retrieval practice). Students were randomly assigned to one of the three learning activities (oral explanation group: $n=40$; written explanation group: $n=39$; retrieval group: $n=36$ ). The dependent variable was students' text comprehension, measured by two knowledge subtests comprising text-based questions and inference-based questions.

To control for potential differences regarding students' prerequisites, we controlled for prior knowledge and reading skills (Ozuru et al., 2009). Additionally, we collected data on students' perceived mental effort, subjective difficulty, and their meta-comprehension judgments.

The size of the recruited sample exceeded the required sample size of 102, as determined by an a-priori power analysis for contrast analyses (GPower, Version 3.1.9.2). Power was set to .80, $\alpha$-error to .05 , and the assumed effect size to $\eta_{\mathrm{p}}^{2}=.05$, as recent studies documented medium effects of explaining modality (Hoogerheide et al., 2016; Lachner et al., 2018).

\subsection{Materials}

The entire experiment was presented in the Qualtrics online survey tool (https://www.qualtrics.com). 
2.2.1 Study Texts. We used two validated texts from the domain of biology from Golke and Wittwer (2018) which systematically differed regarding central dimensions of text difficulty. The first text was about reproduction (low-difficult text) and dealt with types of reproduction (i.e., sexual versus asexual reproduction). The second text was about immunology (high-difficult text) and dealt with immune research based on laboratory mice. The high-difficult text was less cohesive and less concrete than the low-difficult text (see Appendix A, Table A), as indicated by computer-linguistic measures of text difficulty (Berendes et al., 2018). In a pre-study, students ( $N$ $=175$ ) also reported to have invested lower mental effort while reading the low-difficult text than the high-difficult text. Interestingness and perceived simplicity were rated to be comparable across the texts (see Appendix A for the complete pre-study).

2.2.2 Prior knowledge. We used the prior knowledge tests from Golke and Wittwer (2018) which consisted of one test per text. Each test included five open-ended questions which were about general knowledge on the topics (see Appendix B for examples). For each question, students could receive one point, yielding a maximum score of five points per test. Two independent raters coded $20 \%$ of the tests. As the interrater reliability was excellent (ICC > .90, see Koo \& Li, 2016), one rater coded the remaining answers.

2.2.3 Knowledge posttest. For assessing students' learning outcome, we used the knowledge test from Golke and Wittwer (2018). The knowledge posttests comprised of six open text-based questions per text, which aimed at measuring students' basic knowledge of the text (see Appendix B for examples). Furthermore, six open inference-based questions per text measured students' advanced understanding (i.e., situational model, see Appendix B), as they had to combine different aspects and information across the text, and relate them to their prior knowledge. Again, students could receive one point per answer, yielding a maximum score of six 
points for each knowledge test. Two independent raters rated $20 \%$ of the tests. As interrater reliability was excellent $(I C C \geq .90)$, one rater coded the remaining answers.

2.2.4 Perceived mental effort and subjective difficulty. We asked students after the study phase and after the learning activity to rate their invested mental effort ("How much effort did you invest in explaining the material?", Paas, 1992), and subjective difficulty (“How easy was it for you to explain the material?", DeLeeuw \& Mayer, 2008) on a scale from one to nine, as subjective proxies to perceived cognitive load.

2.2.5 Meta-comprehension accuracy. To investigate students' meta-comprehension accuracy, we asked them to make prospective judgments about their expected performance on the knowledge tests ("How confident are you that you can answer questions to the text correctly?", Baars, van Gog, de Bruin, \& Paas, 2017) both after the study phase and after the learning activity on a scale from $0 \%$ (no confidence) to $100 \%$ (absolute confidence; see Schleinschok, Eitel, \& Scheiter, 2017). We operationalized students' meta-comprehension accuracy in terms of bias. Bias refers to the signed difference between students' estimated performance and the actual

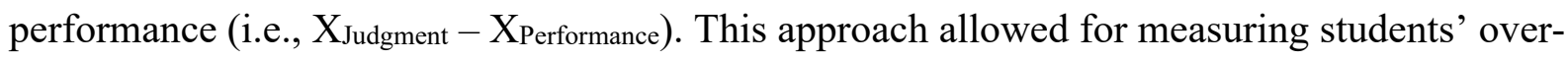
and underestimations of their judged test performance. Positive values indicate an overestimation and negative values indicate an underestimation of the judged performance. A value of zero indicates an accurate judgment.

\subsubsection{Additional control measures.}

2.2.6.1 Reading skills. As students' reading skills could impact their text comprehension (Golke \& Wittwer, 2018; Ozuru et al., 2009), we assessed their reading skills by a German reading and speed comprehension test (LGVT 6-12; Schneider, Schlagmüller, \& Ennemoser, 2007). The LGVT is a speed test that requires students to read a text (text length: 1700 words), as quickly and accurately as possible (duration 4 minutes). The text comprises 25 gaps in which 
students are required to choose which of three different alternative words had to be filled in (for more details see Schneider et al., 2007). Cronbach's alpha was good: $\alpha=.81$.

2.2.6.2 Topic interest. As a further control variable, we measured students' interest in biology with three items (i.e., "I regard biological topics as important", "I am excited about biological topics", "Biological topics are fascinating”, based on Baumert et al., 2008) on a four-point Likert scale from 1 ("I completely disagree") to 4 ("I completely agree"). Cronbach's alpha was excellent: $\alpha=.92$.

2.2.6.3 Self-efficacy in explaining. As a further control variable, we assessed students' selfefficacy in explaining. We used three adapted items (e.g., "I always find ways to explain even difficult contents") from Jerusalem and Schwarzer (1999), and asked students to rate their explaining skills on a four-point Likert scale from 1 ("I completely disagree”) to 4 ("I completely agree"). Cronbach's alpha was good: $\alpha=.79$.

\subsection{Procedure}

The instructor informed the students about the study scope. After providing written consent, the students were randomly assigned to the learning activity conditions (i.e., oral explanation, written explanation, retrieval practice). Afterwards, all students were seated individually in front of a laptop in multi-group sessions $(n=4)$ in two separate rooms. To avoid potential disturbances by other participating students, they wore sound-proof ear protectors during the experiment. The entire study was self-paced. First, the students answered the prior knowledge test, and rated their explaining skills. In the study phase, students read the first text, assessed their invested mental effort and perceived subjective difficulty during reading, and provided a meta-comprehension judgment. In the explaining phase, dependent on learning activity, they randomly provided an oral or a written explanation or retrieved the information of the text. The oral explanation group recorded their explanation via the built-in microphone function of the Windows system. The 
written explanation group wrote an explanation in the Qualtrics environment. Both explaining groups received the following instruction:

Imagine the following scenario: One person could not participate in the study, however, is highly interested in the topic. She has not read anything about the topic yet. She, therefore, asks you to explain the central contents of the text. Please provide her a clear and detailed explanation, so that she can understand the content without additional information.

The control group was engaged in a retrieval practice:

Please retrieve the information of the text.

During the learning activity, all students could take notes on a separate sheet. They were informed that the notes were only available in the learning activity phase but not in the testing phase. Then, the students again provided judgments of their mental effort and subjective difficulty, as well as a meta-comprehension judgment. Afterwards, the students answered the knowledge posttest. Given that students processed two different tests, the procedure was repeated analogously for the second text. The order of the texts was randomized across students. At the end of the study, the students completed the reading skill test and answered the demographic questionnaire (i.e., age, gender, mother language, number of enrolled semesters, interest in topic). The average study time was 90 minutes. After completing the study, the students were debriefed and rewarded with 16 Euros.

\subsection{Analysis and Coding of the Explanations}

2.4.1 Number of personal references. As an indicator of the social presence, we counted the number of personal references per explanation (i.e., "I", “you”, etc.; see Chafe \& Tannen, 1987; Hoogerheide et al., 2016; Lachner et al., 2018). Two independent raters counted the number of 
personal references for $20 \%$ of all explanations. Interrater reliability was excellent, $I C C=1.00$. Therefore, one rater coded the remaining explanations.

2.4.2 Comprehensiveness. As an indicator of the level of comprehensiveness, we counted the number of concepts per explanation (Boshuizen \& Schmidt, 1992). To automatically detect the concepts per explanation, we used CohViz by Burkhart, Lachner, and Nückles (2019) which automatically detects and counts the number of concepts within an explanation by means of natural language processing technologies. For instance, the sentence "After both groups got a vaccination against fever, the group with previous infections showed a different reaction" contains five concepts (marked in italics). Redundant concepts (e.g., "group") were ignored (see also Boshuizen \& Schmidt, 1992, for related approaches). CohViz showed a high precision in comparison to expert raters $(I C C=.93$, see Burkhart et al., 2019).

2.4.3 Number of elaborations. As an indicator of the level of elaboration of the explanations, we counted the number of elaborations per explanation. An elaboration was operationalized as an idea unit which was not covered in the explanation, such as examples, analogies, and own experiences (see also Fiorella \& Kuhlmann, 2019; Lachner et al., 2018). For instance, the sentence "Mice from the pet shop have a completely different immune profile than laboratory mice, similar to the profile of human adults who have already had many diseases, and therefore, have already formed more immune cells" contains one elaboration (marked in italics) since the section was not covered in the study text. Again, two independent raters counted the number of elaborations for $20 \%$ of the explanations. Interrater reliability was excellent $(I C C>.95)$. Thus, one rater coded the remaining explanations.

\section{Results}

We used partial $\eta_{\mathrm{p}}^{2}$ and Cohens' $d$ as effect size measures, qualifying values of $\eta_{\mathrm{p}}^{2}=.01, .06$, .14 , and $d=0.20,0.50,0.80$ as small, medium, and large effects (Cohen, 2013). Additionally, we 
used an alpha level of $\alpha=.05$. In cases of directed hypotheses, we used one-tailed tests (Cho \& Abe, 2011; Furr \& Rosenthal, 2003).

\subsection{Preliminary Analyses}

Analyses showed no significant differences between the learning activity groups concerning age, $F(2,112)=1.54, p=.220, \eta_{\mathrm{p}}^{2}=.027$, and their number of enrolled semesters, ${ }^{1} F(2,101)=$ $0.18, p=.838, \eta_{\mathrm{p}}^{2}=.003$. Students' cognitive and motivational prerequisites were comparable among groups, as the learning activity groups did not differ in reading skills, $F(2,112)=0.27, p=$ $.767, \eta_{\mathrm{p}}^{2}=.005$, regarding prior knowledge of the low-difficult text, $F(2,112)=0.71, p=.496, \eta_{\mathrm{p}}^{2}$ $=.012$, or regarding prior knowledge of the high-difficult text, $F(2,112)=1.26, p=.288, \eta_{\mathrm{p}}^{2}=$ .022. Similarly, there were no differences regarding students' general topic interest, $F(2,112)=$ $1.09, p=.340, \eta_{\mathrm{p}}^{2}=.019$, or their self-efficacy in explaining, $F(2,112)=1.19, p=.310, \eta_{\mathrm{p}}^{2}=$ .021. Additionally, the reading times of the texts were comparable across conditions $\left(F_{\mathrm{S}}<1\right)$, indicating that all learning activity groups invested the same amount of time to process the texts. Regarding the subjective ratings of cognitive $\operatorname{load}^{2}$ of the study phase (i.e., mental effort, subjective difficulty), there was no difference among learning activity groups for the mental effort ratings, $F(2,110)=2.87, p=.061, \eta_{\mathrm{p}}^{2}=.039$, or for the subjective difficulty, $F(2,110)=1.45 p=$ $.240, \eta_{\mathrm{p}}^{2}=.018$. As a further control, we tested whether our data was confounded by potential outliers. Graphical boxplot analyses of the dependent variables revealed that there were three outliers for the text-based questions (see Figure C.1 and C.3 in Appendix C). The outliers were included in the main analyses, as removing them from the sample did not change the findings.

\subsection{Learning-Outcome Hypotheses}

${ }^{1}$ Eleven students did not state their total number of enrolled semesters.

2 Two students did not report their cognitive load. 
We performed two separate mixed factorial ANCOVAs for our two knowledge tests as dependent variables (i.e., text-based questions; inference-based questions). Learning activity (i.e., generating oral vs. written explanations vs. retrieval practice) was the between-students factor and text difficulty (i.e., low-difficult text vs. high-difficult text) was the within-students factor. Additionally, we controlled for students' prior knowledge as covariate.

Regarding the text-based questions, as expected, we found a main effect of text difficulty, $F(1,111)=56.51, p<.001, \eta_{\mathrm{p}}^{2}=.128$, indicating that students performed higher in the lowdifficult text condition than the high-difficult text condition (see Table 1). There was no main effect of learning activity, $F(1,111)=0.78, p=.459, \eta_{\mathrm{p}}^{2}=.007$, and no interaction effect between text difficulty and learning activity, $F(2,111)=0.58, p=.563, \eta_{\mathrm{p}}^{2}=.003$, indicating that the learning activity groups performed comparable on the text-based questions.

Regarding the inference-based questions, we found a significant effect of text difficulty, $F(1$, $111)=15.85, p<.001, \eta_{\mathrm{p}}^{2}=.039$, indicating that students' gained more knowledge from the lowdifficult text than from the high-difficult text (see Table 1). Again, the main effect of learning activity was not significant, $F(1,111)=1.34, p=.261, \eta_{\mathrm{p}}^{2}=.013$. However, in line with our hypothesis, we found a significant interaction between learning activity and text difficulty, $F(2$, $111)=4.67, p=.011, \eta_{\mathrm{p}}^{2}=.023$. To break up the significant interaction, we performed planned contrasts to specifically test our hypotheses. With the first contrast, we tested whether explaining was more beneficial than retrieval practice (i.e., retrieval practice: -2; written explanation: 1; oral explanation: 1). With the second contrast, we tested whether generating oral explanations was more effective than written explanations (i.e., retrieval practice: 0 ; written explanation: -1 ; oral explanation: 1). The contrast analyses revealed that students who generated explanations (oral and written form) outperformed students who simply retrieved the learning material, $t(111)=$ $1.90, p=.030, d=0.36$ (see Table 1 ). More importantly, students who explained orally 
outperformed students who explained in written form, $t(111)=1.81, p=.037, d=0.32$. Together, in line with our hypotheses, we found that the modality of explaining only had an effect for highdifficult text material but not for low-difficult text material. This effect, however, was only significant for higher-order test questions (i.e., inference questions), but not for lower-order test questions (i.e., text-based questions). 
Table 1. Means and Standard Deviations for all Measurements

\begin{tabular}{|c|c|c|c|c|c|c|}
\hline & \multicolumn{3}{|c|}{ Low-difficult text } & \multicolumn{3}{|c|}{ High-difficult text } \\
\hline & Retrieval practice & Written explaining & Oral explaining & Retrieval practice & Written explaining & Oral explaining \\
\hline Prior knowledge & $2.92(1.12)$ & $2.59(1.25)$ & $2.76(1.20)$ & $2.25(0.96)$ & $2.32(1.22)$ & $1.96(0.98)$ \\
\hline \multicolumn{7}{|l|}{ Learning outcomes } \\
\hline Text-based questions & $4.21(1.28)$ & $4.01(1.22)$ & $4.34(1.14)$ & $3.39(1.10)$ & $3.15(1.13)$ & $3.30(1.28)$ \\
\hline Inference-based questions & $3.28(1.03)$ & $2.79(1.12)$ & $3.05(1.21)$ & $2.28(1.08)$ & $2.50(1.17)$ & $2.89(1.25)$ \\
\hline \multicolumn{7}{|l|}{ Perceived Cognitive Load } \\
\hline Perceived Mental Effort & $5.42(1.98)$ & $6.08(2.01)$ & $6.55(1.52)$ & $5.64(2.17)$ & $6.38(1.76)$ & $6.83(1.24)$ \\
\hline Perceived Difficulty & $3.72(2.05)$ & $4.54(2.08)$ & $5.28(1.96)$ & $4.06(2.03)$ & $4.51(2.01)$ & $5.12(1.73)$ \\
\hline Meta-Compr. Accuracy & $0.13(1.50)$ & $0.24(1.38)$ & $-0.46(1.33)$ & $0.54(1.42)$ & $0.90(1.34)$ & $0.60(1.50)$ \\
\hline Reading time & $2.95(2.75)$ & $3.07(2.07)$ & $3.19(1.35)$ & $3.94(2.21)$ & $3.65(2.07)$ & $3.73(1.58)$ \\
\hline \multicolumn{7}{|l|}{ Quality of Explanations } \\
\hline Personal References & & $1.54(1.89)$ & $2.21(3.33)$ & & $0.10(0.45)$ & $0.64(1.46)$ \\
\hline Concepts & & $21.31(7.57)$ & $23.08(6.15)$ & & $18.95(7.63)$ & $25.90(9.12)$ \\
\hline Elaborations & & $2.46(1.68)$ & $2.41(1.53)$ & & $1.00(0.86)$ & $1.39(1.46)$ \\
\hline
\end{tabular}




\subsection{Additional Explorative Analyses}

3.3.1 Effects of explanatory features. To explore the processes underlying the significant modality effect in the high-difficult text condition, we analyzed potential differences regarding the number of personal references, the number of concepts, and the number of elaborations by applying independent $t$-tests. Oral explanations contained more personal references (e.g., "I", "you", etc.) than written explanations, $t(77)=2.20, p=.033, d=0.50$, indicating that the social presence was higher in the oral condition than in the written condition (see Table 1). Regarding the generative processes, oral explanations contained more concepts, as an indicator of the level of comprehensiveness, than written explanations, $t(77)=3.65, p<.001, d=0.83$. However, we did not find a significant difference between written explanations and oral explanations regarding the number of elaborations, $t(77)=1.44, p=.155, d=0.33$. The explorative analyses of the differences of the explanations suggested that the effect of generating oral versus written explanations on students' performance on the inference-based questions mainly resulted, as oral explaining triggered higher amounts of social presence (indicated by the number of personal references) which resulted in more comprehensive explanations (indicated by the number of concepts). To explore this mediation assumption, we conducted a serial mediation analysis. The number of personal references and the number of concepts were the serial mediators, learning activity was the dummy-coded predictor $(1=$ written explanation, $2=$ oral explanation $)$, and students' performance on the inference-based questions (in the high-difficult text) was the dependent variable. We applied a bootstrapping methodology based on Hayes (2013) with 10,000 simulations. Results indicated a significant indirect effect (via number of personal references $\rightarrow$ number of concepts) of $\mathrm{a}_{1} \times \mathrm{d}_{21} \times \mathrm{b}_{2}=.09,95 \% \mathrm{CI}[.010, .313]$, as zero was not included in the confidence interval (see Figure 1, for the full mediation model). There were no direct effects via social presence or comprehensiveness. The significant serial mediation effect suggests that the 
superiority of generating oral versus written explanations resulted, as students had higher levels of social presence during oral explaining (as indicated by the number of personal references), which inclined them to provide more comprehensive explanations and include more concepts in their explanations.

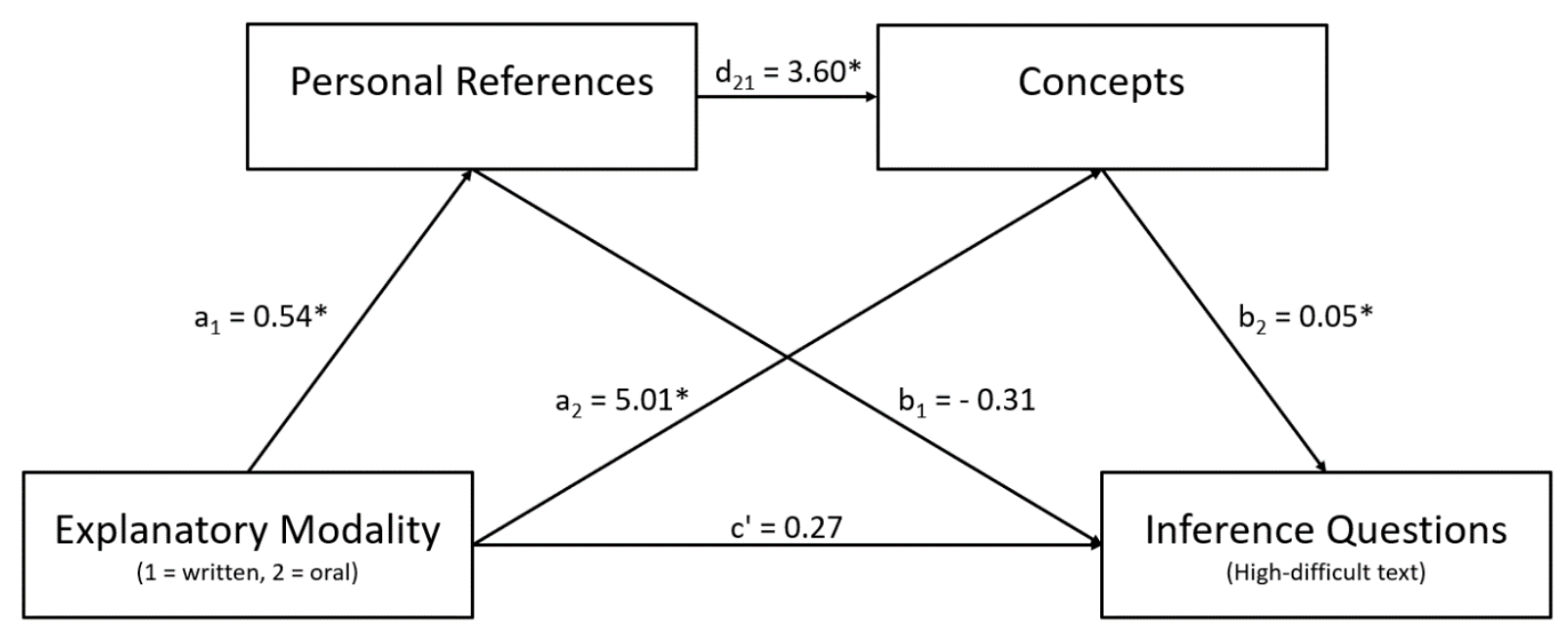

Figure 1. Serial mediation analysis regarding concepts in explanations. ${ }^{*} p<.05$.

3.3.2 Effects on students' mental effort. To investigate students' mental effort during the learning activity (i.e., explaining or retrieving), we conducted a repeated measures ANOVA ${ }^{3}$ with students' mental effort ratings as a dependent variable, learning activity (i.e., generating oral vs. written explanations vs. retrieval practice) as a between-students factor, and text difficulty (i.e., low-difficult text vs. high-difficult text) as a within-students factor. Results showed no main effect of text difficulty, $F(1,110)=2.21, p=.140, \eta_{\mathrm{p}}^{2}=.004$, indicating that processing the texts after administering the subsequent learning activities (i.e., generating oral vs. written

\footnotetext{
${ }^{3}$ Two students did not report their cognitive load.
} 
explanations vs. retrieval practice) was perceived as comparably effortful across conditions of text difficulty. However, we found a main effect of learning activity, $F(2,110)=5.15, p=.007$, $\eta_{\mathrm{p}}^{2}=.069$. The interaction between learning activity and text difficulty was not significant, $F(2$, $110)=.080, p=.923, \eta_{\mathrm{p}}^{2}<.001$. Post-hoc comparisons (Scheffe) indicated that students reported more effort during oral explaining than retrieving the contents $(p<.001$, see Table 1$)$. There were no significant differences between generating oral or written explanations $(p=.251)$ or between writing explanations and retrieving the contents $(p=.057)$.

3.3.3 Effects on students' subjective difficulty. Similar to previous analyses, a repeated measures $\mathrm{ANOVA}^{4}$ revealed no significant main effect of text difficulty, $F(1,110)=0.12, p=$ $.733, \eta_{\mathrm{p}}^{2}<.001$, but a main effect of the learning activity, $F(2,110)=7.00, p=.001, \eta_{\mathrm{p}}^{2}=.080$. Again, the interaction effect was not significant, $F(2,110)=0.38, p=.684, \eta_{\mathrm{p}}^{2}<.001$.

Similar to the mental effort ratings, post-hoc tests revealed that students perceived oral explaining as more difficult than retrieving the contents $(p<.001$, see Table 1$)$. Again, there were no differences between generating oral versus written explanations $(p=.050)$ or between writing explanations and retrieving the contents $(p=.140)$.

3.3.4 Meta-comprehension accuracy. To investigate whether students' meta-comprehension accuracy increased after the explaining task, we conducted an ANCOVA with students' metacomprehension accuracy after the learning activity as a dependent variable, the low- and the highdifficult text as a within-students factor, and type of learning activity (i.e., generating oral vs. written explanation vs. retrieval practice) as a between-students factor. Additionally, we controlled for students' meta-comprehension accuracy during the study phase to control for potential intra-individual differences (see Hertzog, Hines, \& Touron, 2013; Lachner et al., 2019).

${ }^{4}$ Two students did not report their cognitive load. 
Results demonstrated a main effect of learning activity, $F(2,111)=9.05, p<.001, \eta_{\mathrm{p}}^{2}=.032$, and a main effect of text difficulty, $F(1,111)=41.11, p<.001, \eta_{\mathrm{p}}^{2}=.039$, indicating that students rated their comprehension more precisely in the low-difficult text condition than in the high-difficult text condition (see Table 1). There was no interaction between learning activity and text difficulty, $F(2,111)=0.04, p=.963, \eta_{\mathrm{p}}^{2}=.000$. Post-hoc tests showed a significant difference between students who explained orally and students who wrote an explanation $(p<$ .001). Students who explained orally estimated their comprehension more accurately (see Table 1). Additionally, there was a significant difference between students who generated an oral explanation and students who retrieved the material $(p=.026)$. Again, explaining orally resulted in better meta-comprehension accuracy. However, results indicated no effect between students who wrote an explanation and students who retrieved the material $(p=.446)$.

\section{Discussion}

In the current study we investigated whether the effect of explaining modality depends on the level of text difficulty of the studying material. As expected, we showed that explaining modality mainly affected students' performance when they learned from high-difficult learning material, as students who generated oral explanations outperformed students who generated written explanations. Students who simply did retrieval practice showed the lowest performance. For the low-difficult learning material, the three learning activities were comparably effective, since there were no significant differences among learning activities. Thus, text difficulty moderated the effect of explaining modality on students' learning. Whereas low-difficult learning material may suffice to support students in constructing a coherent representation of the text (Berendes et al., 2018; McNamara, 2013), which may make subsequent learning activities obsolete, for the highdifficult materials, the addition of a generative learning activity was a necessary precondition to help students establish a coherent understanding of the text (see also Roelle \& Nückles, 2019, for 
related findings). This effect was more pronounced for explaining orally than for writing explanations. Therefore, our findings highlight that the need for additional learning activities depends on the quality of previous learning materials.

Second, we showed that the superior effects of generating oral versus written explanations occurred, as oral explaining triggered higher levels of social presence during explaining. The increased social presence resulted in more generative processes, as indicated by more comprehensive explanations in the oral explaining condition. Therefore, our findings corroborate the social presence hypothesis (Hoogerheide et al., 2016, 2019; Lachner et al., 2018) that effects of explaining are not only due to generative processes but also due to increases of social presence. Our mediation analyses helped get a better understanding of the cognitive mechanisms of increases of social presence. For instance, Hoogerheide et al. (2019) suggested both, a direct effect of social presence, due to increases of arousal, and an indirect effect of social presence by affecting the generative processes that take place during explaining. Our findings rather support the indirect social presence hypothesis, as we only obtained a significant serial mediation effect of the explaining modality on students' learning via social presence and comprehensiveness of explanations but not a direct effect solely via social presence.

Our explorative analyses regarding students' meta-comprehension accuracy showed a further interesting detail. The findings showed, that the modality of explaining accounted for the accuracy of their meta-comprehension (see Fukaya, 2013). Oral explaining generally resulted in more accurate judgements compared to retrieval practice but also compared to writing explanations. This finding complements previous research which demonstrated that explaining supports students' meta-comprehension accuracy (Fukaya, 2013; Lachner et al., 2019) but adds to previous research, as it shows that improved meta-comprehension accuracy depends on the modality of explaining. 
An unexpected finding was that only the comprehensiveness of the explanation but not the elaboration of the explanations mediated the effect of explaining modality on students' learning outcomes. This finding is surprising, as recent studies documented the central role of elaborations during explaining (Fiorella \& Kuhlmann, 2019; Lachner et al., 2018). We attribute this finding to the fact that students processed biological contents, which made it difficult to generate elaborations such as examples during explaining. This was also reflected in the medium number of elaborations in the explaining conditions (see Table 1).

\subsection{Study Limitations and Future Research}

One potential caveat refers to the fact that we realized a within-students design, which increased test power but was likely more vulnerable for potential carry-over effects. To counteract carry-over effects, we provided the students with a random order of texts, which varied only regarding text difficulty, but not regarding potential other factors such as interestingness (see Appendix A). This procedure should reduce the risk of potential confounding factors. Nevertheless, further research should replicate our findings, for instance, by realizing a between-students design, in which text difficulty and learning activity are manipulated as between-students factors.

Another limitation is that we only measured the quality of explanations as a proxy for the underlying processes of the modality effect (see Fiorella \& Kuhlmann, 2019; Lachner et al., 2018). However, assessing students' products of explaining (i.e., comprehensiveness and elaborations) - more importantly for the written explanation condition — does not provide an "online" measure of the underlying processes of the explaining modality effect. For instance, it could be the case that, particularly in the written explanation condition, students could have elicited more elaborations than they actually realized in their explanations. Such an effect can be expected, as writing typically involves more conscious decisions about inserting or withholding 
information than providing oral explanations (Lachner et al., 2018; Nückles et al., 2009). Thus, future studies should include online-measures, such as think-aloud protocols (Lachner et al., 2019) or log-file-data to more directly assess the underlying cognitive mechanisms of explaining.

Finally, we have to note that we conducted our study in a laboratory setting. Therefore, more research is needed in applied contexts and different study populations to test the robustness of our findings (see Hoogerheide et al., 2019, for examples).

\subsection{Conclusion}

Our findings demonstrate that explaining to fictitious others is a beneficial strategy to support students' learning, particularly when they are required to learn from difficult learning materials. Furthermore, our findings suggest that the effectiveness of explaining depends on the modality of explaining. Due to differences in perceived social presence between written and oral explaining, providing written and oral explanations might be both appropriate if the primary learning goal is the acquisition of basic knowledge. However, if the main goal is to enable students to construct coherent higher-order knowledge, generating oral explanations may best enhance students' learning. 


\section{References}

Baars, M., van Gog, T., de Bruin, A., \& Paas, F. (2017). Effects of problem solving after worked example study on secondary school children's monitoring accuracy. Educational Psychology, 37(7), 810-834. doi:10.1080/01443410.2016.1150419

Baumert, J., Blum, W., Brunner, M., Dubberke, T., Jordan, A., Klusmann, U., Krauss, S., Kunter, M., Löwen, K., Neubrand, M., \& Tsai, Y.-M. (2008). Professionswissen von Lehrkräften, kognitiv aktivierender Mathematikunterricht und die Entwicklung von mathematischer Kompetenz (COACTIV): Dokumentation der Erhebungsinstrumente. Berlin: Max-PlanckInstitut für Bildungsforschung.

Berendes, K., Vajjala, S., Meurers, D., Bryant, D., Wagner, W., Chinkina, M., \& Trautwein, U. (2018). Reading demands in secondary school: Does the linguistic complexity of textbooks increase with grade level and the academic orientation of the school track? Journal of Educational Psychology, 110(4), 518-543. doi:10.1037/edu0000225

Boshuizen, H. P. A., \& Schmidt, H. G. (1992). On the role of biomedical knowledge in clinical reasoning by experts, intermediates and novices. Cognitive Science, 16(2), 153-184.

Burkhart, C., Lachner, A., \& Nückles, M. (2019). Assisting students' writing with computerbased concept map feedback: A validation study of the CohViz Feedback System. Manuscript submitted for publication.

Chafe, W., \& Tannen, D. (1987). The relation between written and spoken language. Annual Review of Anthropology, 16(1), 383-407.

Chin, D. B., Dohmen, I. M., Cheng, B. H., Oppezzo, M. A., Chase, C. C., \& Schwartz, D. L. (2010). Preparing students for future learning with teachable agents. Educational Technology Research and Development, 58(6), 649-669. doi:10.1007/s11423-010-9154-5 
Cho, H.-C., \& Abe, S. (2011). Is two-tailed testing for directional research hypotheses tests legitimate? Journal of Business Research, 66(9), 1261-1266. doi:10.1016/j.jbusres.2012.02.023

Cohen, J. (2013). Statistical power analysis for the behavioral sciences (2nd ed.). Hoboken: Taylor and Francis.

DeLeeuw, K. E., \& Mayer, R. E. (2008). A comparison of three measures of cognitive load: Evidence for separable measures of intrinsic, extraneous, and germane load. Journal of Educational Psychology, 100(1), 223-234. doi:10.1037/0022-0663.100.1.223

Fiorella, L., \& Kuhlmann, S. (2019). Creating drawings enhances learning by teaching. Journal of Educational Psychology. doi:10.1037/edu0000392

Fiorella, L., \& Mayer, R. E. (2013). The relative benefits of learning by teaching and teaching expectancy. Contemporary Educational Psychology, 38(4), 281-288.

doi:10.1016/j.cedpsych.2013.06.001

Fiorella, L., \& Mayer, R. E. (2014). Role of expectations and explanations in learning by teaching. Contemporary Educational Psychology, 39(2), 75-85.

doi:10.1016/j.cedpsych.2014.01.001

Fukaya, T. (2013). Explanation generation, not explanation expectancy, improves metacomprehension accuracy. Metacognition and Learning, 8(1), 1-18. doi:10.1007/s11409012-9093-0

Furr, R. M., \& Rosenthal, R. (2003). Repeated-measures contrasts for "multiple-pattern" hypotheses. Psychological Methods, 8(3), 275-293. doi:10.1037/1082-989X.8.3.275

Golke, S., \& Wittwer, J. (2018). High-performing readers underestimate their text comprehension: Artifact or psychological reality? Manuscript submitted for publication. 
Hayes, A. F. (2013). Introduction to mediation, moderation, and conditional process analysis: A regression-based approach. New York: Guilford Press.

Hertzog, C., Hines, J. C., \& Touron, D. R. (2013). Judgments of learning are influenced by multiple cues in addition to memory for past test accuracy. Archives of Scientific Psychology, 1(1), 23-32. doi:10.1037/arc0000003

Hinds, P. J., Patterson, M., \& Pfeffer, J. (2001). Bothered by abstraction: The effect of expertise on knowledge transfer and subsequent novice performance. Journal of Applied Psychology, 86(6), 1232-1243. doi:10.1037/0021-9010.86.6.1232

Hoogerheide, V., Deijkers, L., Loyens, S. M.M., Heijltjes, A., \& van Gog, T. (2016). Gaining from explaining: Learning improves from explaining to fictitious others on video, not from writing to them. Contemporary Educational Psychology, 44-45, 95-106. doi:10.1016/j.cedpsych.2016.02.005

Hoogerheide, V., Loyens, S. M.M., \& van Gog, T. (2014). Effects of creating video-based modeling examples on learning and transfer. Learning and Instruction, 33, 108-119. doi:10.1016/j.learninstruc.2014.04.005

Hoogerheide, V., Visee, J., Lachner, A., \& van Gog, T. (2019). Generating an instructional video as homework activity is both effective and enjoyable. Learning and Instruction, 64, 1-13. doi:10.1016/j.learninstruc.2019.101226

Jerusalem, M., \& Schwarzer, R. (1999). Allgemeine Selbstwirksamkeitserwartung. In R. Schwarzer \& M. Jerusalem (Eds.), Skalen zur Erfassung von Lehrer- und Schülermerkmalen: Dokumentation der psychometrischen Verfahren im Rahmen der Wissenschaftichen Begleitung des Modellversuchs Selbstwirksame Schulen (pp. 13-14). Berlin. 
Jucks, R. (2001). Was verstehen Laien? Die Verständlichkeit von Fachtexten aus der Sicht von Computer-Experten. Münster: Waxmann.

Kiewra, K. A. (2005). Learn how to study and SOAR to success. Upper Saddle River: Pearson, Prentice Hall.

Kobayashi, K. (2018). Learning by preparing-to-teach and teaching: A meta-analysis. Japanese Psychological Research, 61(3), 1-12. doi:10.1111/jpr.12221

Koo, T. K., \& Li, M. Y. (2016). A guideline of selecting and reporting intraclass correlation coefficients for reliability research. Journal of Chiropractic Medicine, 15(2), 155-163. doi:10.1016/j.jcm.2016.02.012

Lachner, A., Backfisch, I., Hoogerheide, V., van Gog, T., \& Renkl, A. (2019). Timing matters! Explaining between study phases enhances students' learning. Journal of Educational Psychology. Advance online publication. doi:10.1037/edu0000396

Lachner, A., Ly, K.-T., \& Nückles, M. (2018). Providing written or oral explanations? Differential effects of the modality of explaining on students' conceptual learning and transfer. Journal of Experimental Education, 86(3), 344-361. doi:10.1080/00220973.2017.1363691

Lachner, A., \& Nückles, M. (2015). Bothered by abstractness or engaged by cohesion? Experts' explanations enhance novices' deep-learning. Journal of Experimental Psychology: Applied, 21(1), 101-115. doi:10.1037/xap0000038

McNamara, D. S. (2013). The epistemic stance between the author and reader: A driving force in the cohesion of text and writing. Discourse Studies, 15(5), 579-595. doi:10.1177/1461445613501446 
McNamara, D. S., Kintsch, E., Songer, N. B., \& Kintsch, W. (1996). Are good texts always better? Interactions of text coherence, background knowledge, and levels of understanding in learning from text. Cognition and Instruction, 14(1), 1-43. doi:10.1207/s1532690xci1401_1

McNamara, D. S., \& Knitsch, W. (2009). Learning from texts: Effects of prior knowledge and text coherence. Discourse Processes, 22(3), 247-288.

Okita, S. Y., \& Schwartz, D. L. (2013). Learning by teaching human pupils and teachable agents: The importance of recursive feedback. Journal of the Learning Sciences, 22(3), 375-412. doi:10.1080/10508406.2013.807263

O'Reilly, T., \& McNamara, D. S. (2007). Reversing the reverse cohesion effect: Good texts can be better for strategic, high-knowledge readers. Discourse Processes, 43(2), 121-152. doi:10.1080/01638530709336895

Ozuru, Y., Briner, S., Best, R., \& McNamara, D. S. (2010). Contributions of self-explanation to comprehension of high- and low-cohesion texts. Discourse Processes, 47(8), 641-667. doi:10.1080/01638531003628809

Ozuru, Y., Dempsey, K., \& McNamara, D. S. (2009). Prior knowledge, reading skill, and text cohesion in the comprehension of science texts. Learning and Instruction, 19(3), 228-242. doi:10.1016/j.learninstruc.2008.04.003

Paas, F. (1992). Training strategies for attaining transfer of problem-solving skill in statistics: A cognitive-load approach. Journal of Educational Psychology, 84(4), 429-434. doi:10.1037/0022-0663.84.4.429

Palinscar, A. S., \& Brown, A. L. (1984). Reciprocal teaching of comprehension-fostering and comprehension-monitoring activities. Cognition and Instruction, 1(2), 117-175. doi:10.1207/s1532690xci0102_1 
Plötzner, R., Dillenbourg, P., Praier, M., \& Traum, D. (1999). Learning by explaining to oneself and to others. In P. Dillenbourg (Ed.), Collaborative-learning: Cognitive and Computational Approaches (pp. 103-121). Oxford: Elsevier.

Roediger III, H. L. \& Karpicke, J. D. (2006). The power of testing memory: Basic research and implications for educational practice. Perspectives on Psychological Science, 1(3), 181-210.

Roelle, J., \& Nückles, M. (2019). Generative learning versus retrieval practice in learning from text: The cohesion and elaboration of the text matters. Journal of Educational Psychology. Advance online publication. doi:10.1037/edu0000345

Roscoe, R. D. (2014). Self-monitoring and knowledge-building in learning by teaching. Instructional Science, 42(3), 327-351. doi:10.1007/s11251-013-9283-4

Roscoe, R. D., \& Chi, M. T. H. (2008). Tutor learning: The role of explaining and responding to questions. Instructional Science, 36(4), 321-350. doi:10.1007/s11251-007-9034-5

Schleinschok, K., Eitel, A., \& Scheiter, K. (2017). Do drawing tasks improve monitoring and control during learning from text? Learning and Instruction, 51, 10-25. doi:10.1016/j.learninstruc.2017.02.002

Schneider, W., Schlagmüller, M., \& Ennemoser, M. (2007). LGVT 6-12: Lesegeschwindigkeitsund -verständnistest für die Klassen 6-12. Göttingen: Hogrefe.

Wharton, C., \& Kintsch, W. (1991). An overview of the construction-integration model: A theory of comprehension as a foundation for a new cognitive architecture. SIGART Bulletin, 2(4), 169-173. 
Appendix A. Pre-study

We empirically investigated the linguistic differences among text-conditions in a pre-study as a further manipulation check. We invited university students (who did not participate in the main study) via the university mailing list to judge the text difficulty of the two texts by means of an online questionnaire. The findings are based on a sample of 175 students (42 students were excluded, as they did not complete the pre-study or German was not their mother language). The students were comparable to the main study (mean age: 22.65 years, $S D=3.66,79 \%$ female). Students randomly read the high- or the low-difficult text, and judged the subjective difficult, and their invested mental effort (see Material section of main study) on a scale from one to nine, as subjective proxies to perceived cognitive load. Additionally, the students rated general characteristics of the text by means of a questionnaire of Jucks (2001) comprising the following dimensions: a) simplicity (5 items, for instance, "The text contains short and easy sentences", Cronbach's $\alpha$ low-difficult text $=.56$, Cronbach's $\alpha$ high-difficult text $=.67)$; b) cohesion (6 items, for instance, "The text has a consistent story line", Cronbach's $\alpha$ low-difficult text $=.84$, Cronbach's $\alpha$ highdifficult text $=.87) ; \mathrm{c}$ ) conciseness (6 items, for instance, "The text is limited to the essential information", Cronbach's $\alpha$ low-difficult text $=.46$, Cronbach's $\alpha$ high-difficult text $=.61)$;) interestingness (4 items, for instance, “The text is interesting”, Cronbach's $\alpha$ low-difficult text $=.84$, Cronbach's $\alpha$ high-difficult text $=.78$ ). Then, the students read the second text and answered identical questions.

In line with the linguistic analyses, students invested more mental effort while reading the high-difficult text, $t(174)=2.60, p=.010, d=0.15$. Additionally, they stated higher difficulties when reading the high-difficult text compared to the low-difficult text, $t(174)=3.17, p=.002, d$ $=0.31$. However, we did not find significant differences between the low-difficult and the highdifficult text for perceived simplicity, $t(174)=0.14, p=.890, d=0.01$, perceived text cohesion, 
$t(174)=-1.08, p=.281, d=-0.11$, and interestingness of the texts, $t(174)=-.07, p=.287, d=$ 0.11 , indicating that apparently students were not directly aware of the linguistic differences between both texts (see descriptive statistics in Table A). Interestingly, students rated the highdifficult text as less concise than the low-difficult text, $t(174)=-3.36, p=.001, d=-0.29$, although both texts had a comparable text length (words low-difficult text: 380 , words high-difficult text: 397). These findings may be indicative of the fact that students had to invest more mental effort during reading the high-difficult text which resulted in different perceptions of text length (i.e., conciseness).

Table A. Features of both learning texts

\begin{tabular}{|c|c|c|}
\hline Features & Low-difficult text & High-difficult text \\
\hline Topic & Reproduction & Immunology \\
\hline Genre & Informative factual text & Informative factual text \\
\hline \multicolumn{3}{|l|}{ Linguistic features } \\
\hline Number of words & 380 & 397 \\
\hline Number of sentences & 25 & 26 \\
\hline Words per sentence & 15.2 & 15.3 \\
\hline Syntactical structure & 9.12 & 9.79 \\
\hline Global cohesion & 0.16 & 0.08 \\
\hline Word concreteness & 15.5 & 9.5 \\
\hline Flesh-Index & 46 & 41 \\
\hline \multicolumn{3}{|c|}{ Perception of the text (Pre-Study) } \\
\hline Mental effort & $4.72(S D=1.91)$ & $5.01(S D=1.94)$ \\
\hline Subjective difficulty & $2.92(S D=1.78)$ & $3.48(S D=1.84)$ \\
\hline Simplicity & $3.56(S D=0.61)$ & $3.57(S D=0.70)$ \\
\hline Cohesion & $3.70(S D=0.72)$ & $3.61(S D=0.80)$ \\
\hline Conciseness & $2.97(S D=0.53)$ & $2.81(S D=0.55)$ \\
\hline Interestingness & $3.41(S D=0.88)$ & $3.50(S D=0.83)$ \\
\hline
\end{tabular}


Note. Linguistic features were calculated according to McNamara (2013), Berendes et al. (2018), and Lachner and Nückles (2015). Perception of the text was rated by 175 students (see Materials section). 
Appendix B. Item examples of knowledge tests

\begin{tabular}{lll}
\hline & \multicolumn{1}{c}{ Low-difficult text } & \multicolumn{1}{c}{ High-difficult text } \\
\hline Prior knowledge questions & How does offspring arise from asexual & Why can viruses be dangerous for \\
& reproduction? & humans? \\
Text-based questions & What is the disadvantage of sexual & What is the main concern against \\
& reproduction? & research with laboratory mice? \\
Inference-based questions & How would the health of animals be & How can the results help to clarify the \\
& affected if they produced offspring with & basic problems of immune research \\
& asexual reproduction? & with mice?
\end{tabular}


Appendix C. Boxplots of Posttest Performance per Condition

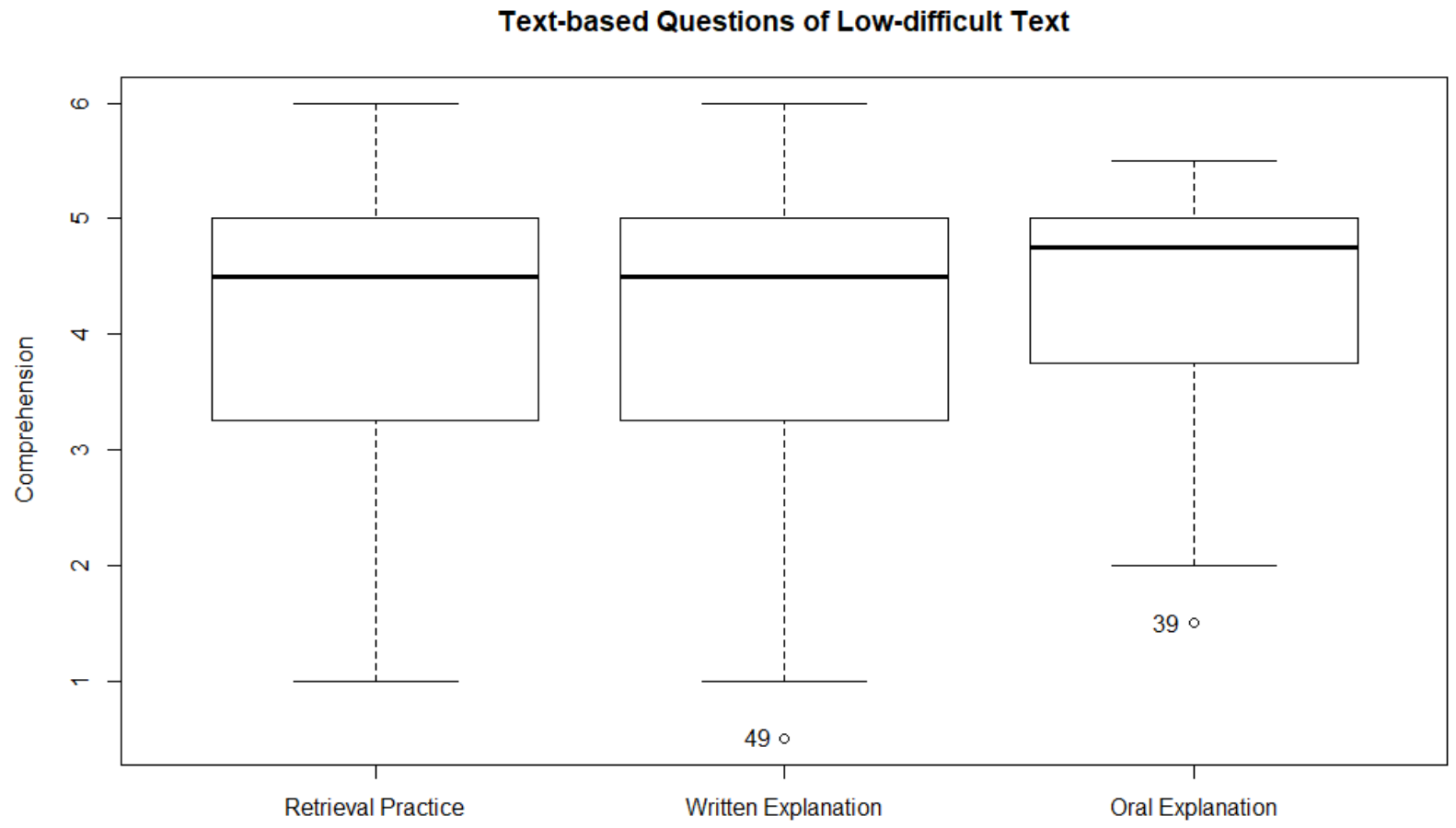

Figure C.1. 
Inference-based Questions of Low-difficult Text

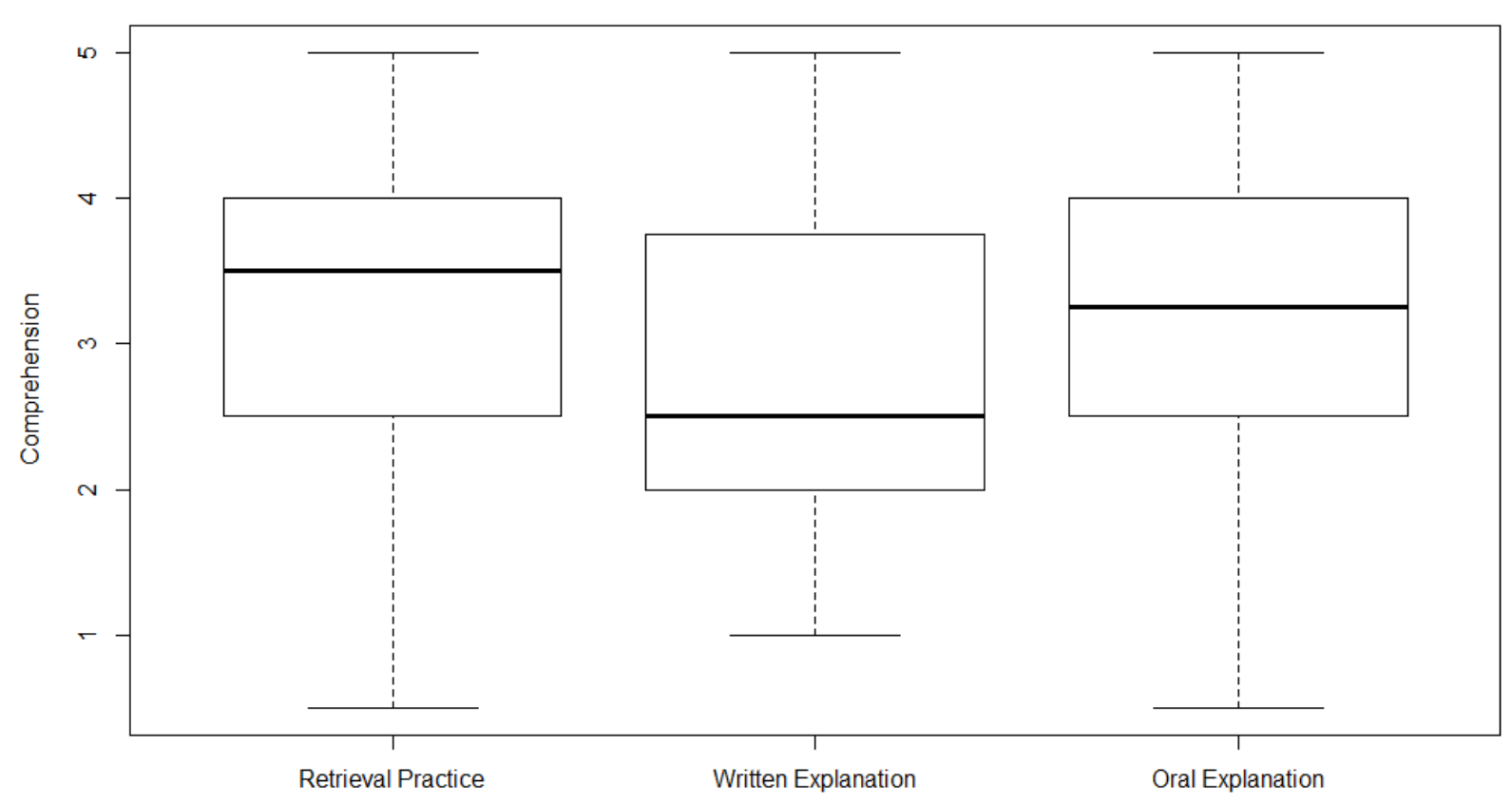

Figure C.2. 
Text-based Questions of High-difficult Text

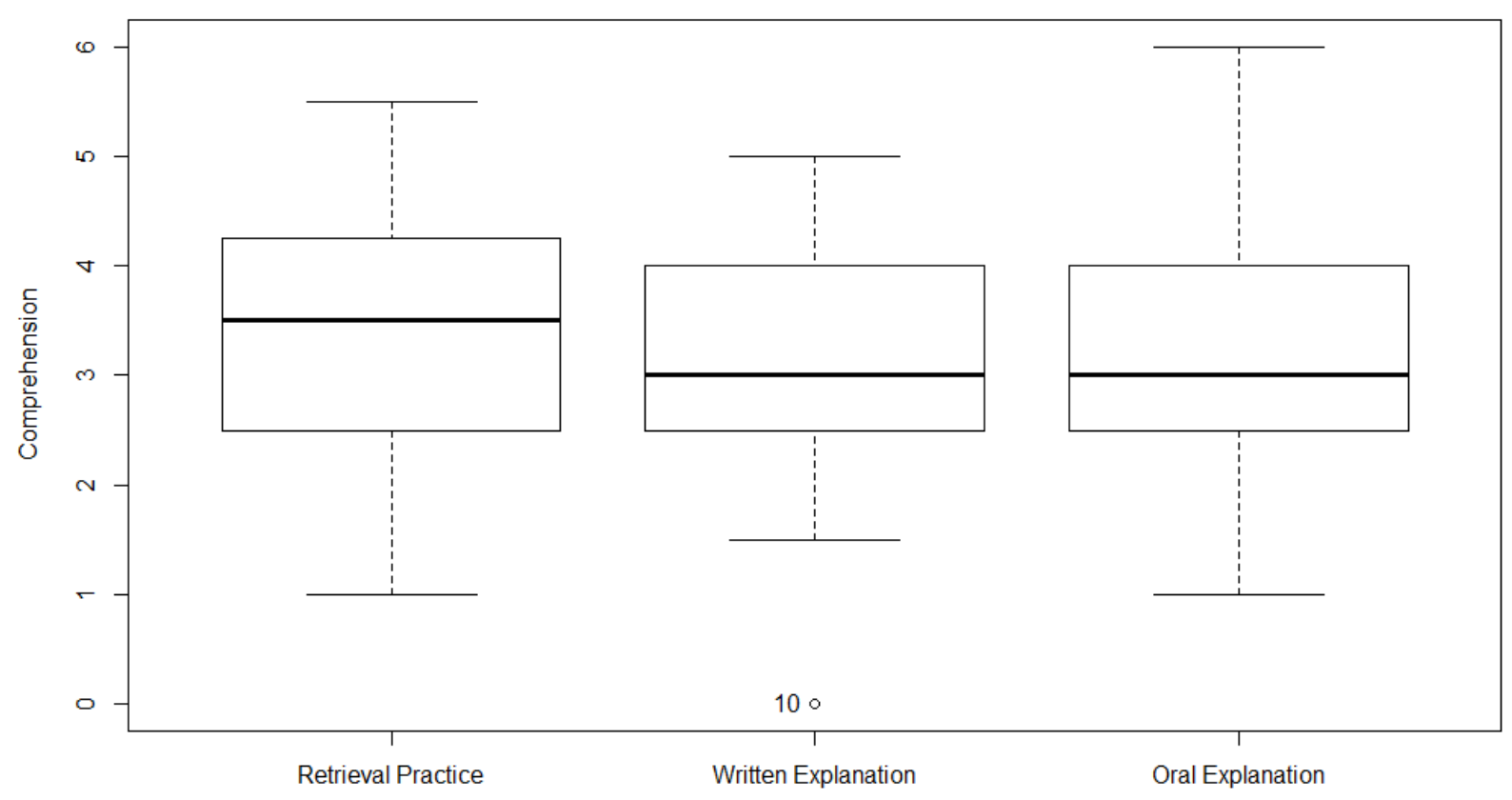

Figure C.3. 
Inference-based Questions of High-difficult Text

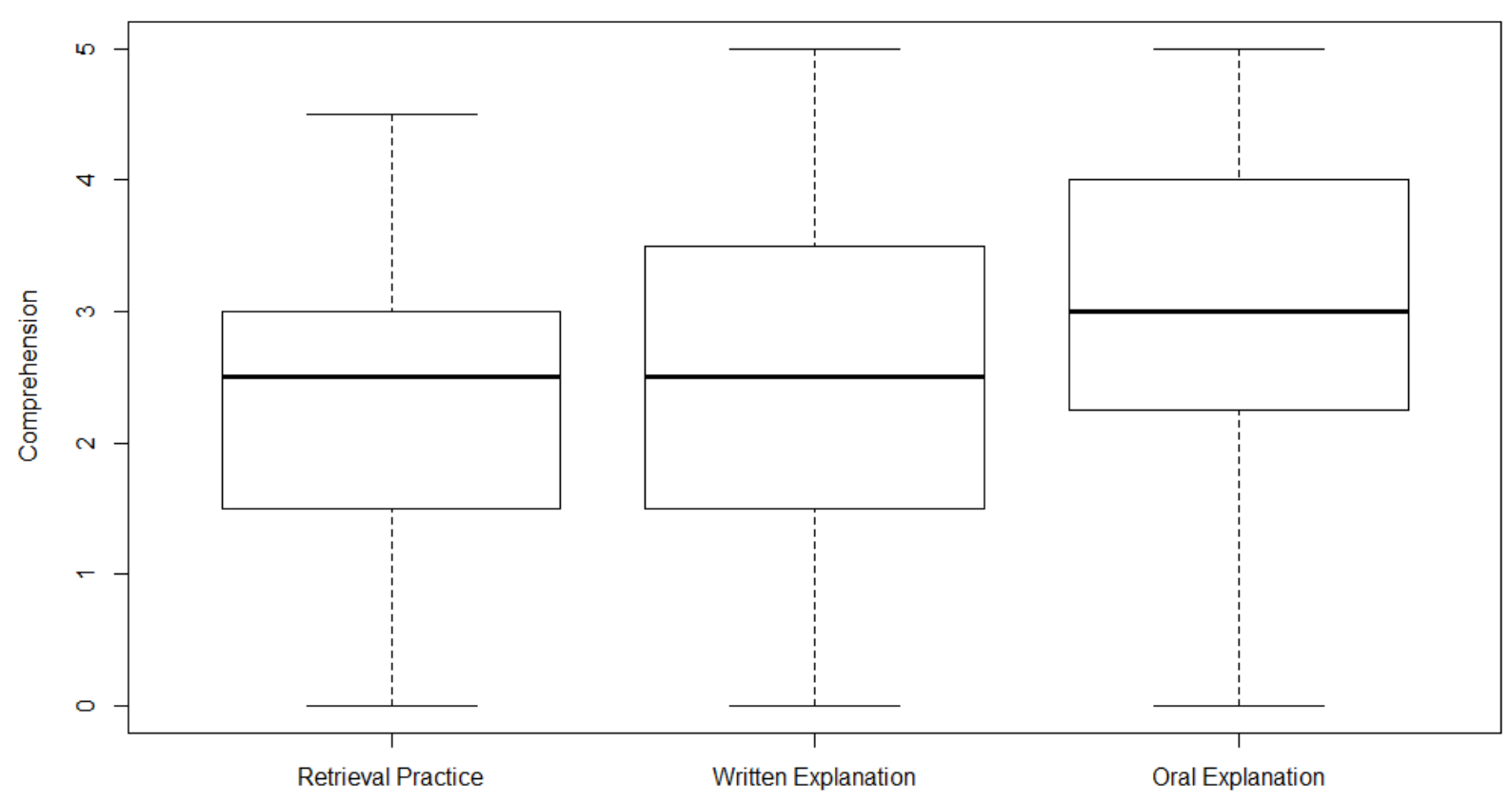

Figure C.4. 